\title{
Capacidades institucionales en el desarrollo regional para los países de América Latina
}

\section{Institutional capacities in Regional Development for the Latin American countries}

Víctor Ramiro Fernández, María Cecilia Güemes, Juan Pablo Magnin y José Ignacio Vigil (2006), CAPACIDADES ESTATALES Y DESARROLLO REGIONAL. REALIDADES Y DESAFIOS PARA AMÉRICA LATINA, UnIVERSI-

dad Nacional del Litoral, Santa Fe, Argentina, 298 Pp., isbn

$$
\text { 978-987-508-669-2. }
$$

El eje central del texto que reseñamos, es la firme necesidad de rescatar el papel estratégico adjudicado al Estado como primer motor del desarrollo. En los estudios sobre el territorio, el análisis del Estado y sus formas de intervención son esenciales para entender sus transformaciones. En este caso, la originalidad del debate se orienta mediante un enfoque transdisciplinario y una sólida argumentación, que proporcionan las bases sobre la urgencia de generar capacidades estatales para fortalecer a los Estados latinoamericanos. El contexto en el que se aborda el análisis es el proceso de globalización, aunado a las políticas de orientación neoliberal surgidas a partir del Consenso de Washington, y que fueron acogidas por diferentes gobiernos de la región. El análisis empírico se fundamenta en un estudio de caso desarrollado en la provincia de Santa Fe, Argentina, específicamente en el sector productivo industrial, con la intención de caracterizar el conjunto de intervenciones orientadas a reorganizar las capacidades económicas y tecnológicas para dar centralidad a una estrategia de articulación multiescalar y un desarrollo regional integral e integrador.

El texto se estructura en tres grandes vertientes: la primera se enfoca a reintegrar justamente el rol que le concierne al Estado, de acuerdo con sus capacidades institucionales, relacionando la escasa fundamentación de su papel en los enfoques regionalistas desarrollados en las últimas décadas. El principio integrador de esta primera parte responde a la pregunta: ¿cuáles son los fundamentos de fondo que se usaron como herramientas o armas para colocar a las regiones como las plataformas de desarrollo más apropiadas en el escenario de la globalización? La segunda vertiente se relaciona con la fase empírica, con el traslado de los principales aportes teórico-conceptuales y metodológicos analizados en la pri- 
mera parte y la urgencia de analizar las capacidades estatales en determinados escenarios regionales. Es decir, ¡cómo han evolucionado en Argentina, durante la década de los noventa, las capacidades institucionales de las oficinas estratégicamente prioritarias del Estado regional (OEPER), que articulan la producción, el territorio y la industria, así como la organización de los actores público y privado en el ámbito regional? Por su parte, la tercera y última vertiente se vincula con la integración de un conjunto de lineamientos que recolocan al Estado como motor del desarrollo, o bien, ¿en qué forma y en qué campos de intervención el Estado debe trasladarse para superar el fragmentalismo y la fuerte propensión a potenciar los desequilibrios económicos territoriales?

De esta forma, y en referencia al primer propósito del libro, se argumenta que los enfoques sobre el desarrollo regional centrados en las ventajas estáticas fundamentados en la escuela de California y la nueva geografía económica, asociados al desarrollo de las ventajas dinámicas basadas en los sistemas regionales de innovación y la perspectiva centrada en las cadenas de valor, tienen auge en explicar una trayectoria de regiones exitosas, localizadas en las zonas más desarrolladas de determinados países, por ejemplo, los distritos industriales italianos, la industria cinematográfica de Hollywood, los distritos del calzado en Valencia, o bien las expresiones high tech de Syllicon Valley, Cambridge o Grenoble (p. 17). Sin embargo, los autores destacan la importancia de incorporar en la perspectiva regionalista, y específicamente en los países de América Latina, dos aportes teórico-empíricos originados en la sociología, la ciencia política y la economía política comparada: los legados políticos y los aprendizajes sociales como condicionantes de las nuevas formas de organización en red, y los límites derivados de los procesos de causación acumulativa que fortalecen a determinados centros en detrimento de otros, para formar parte de un proceso integrador de desarrollo regional y, de alguna manera, revertir la cristalización de las asimetrías territoriales.

Desde esta perspectiva, se parte del supuesto de que el Estado como primer motor institucional, estimula y condiciona el comportamiento de los diversos actores regionales para orientarlos hacia nuevas lógicas de coordinación en red, no obstante, la inapropiada incorporación en los enfoques sobre el desarrollo generados en la región, donde se le relega al rol de una agencia más, se continuó sin otorgarle especificidad institucional, para capitalizar legados y redefinir aprendizajes.

Así, se propone recuperar el papel del Estado, justamente es su calidad de primer motor en la reelaboración de aprendizajes orientados a generar un desarrollo regional de carácter integral, por tanto, conviene reconocer dos grandes campos: la nueva morfología y los nuevos contenidos deman- 
dados a la intervención estatal, así como los atributos de la estatidad regional, traducidos como capacidades estatales de los gobiernos regionales.

Se explica que en el nuevo contexto de la acumulación flexible y dada la pluralidad de actores sociales, la forma de coordinación por redes ha generado las condiciones necesarias de integración, flexibilidad y dinamismo para construir tanto las actividades estáticas como dinámicas, basadas principalmente en la innovación, redefiniendo así tanto la acción estatal como la morfología del mismo Estado. En términos territoriales la transformación, o bien, la modificación de la conducción del Estado, lleva a una definición de los gobiernos locales y regionales, pero fundamentados en un papel protagónico. Sin embargo, la recolocación del Estado desde su dimensión regional como instrumento estratégico en la potenciación del desarrollo, no se agota en el análisis de la redefinición en sus patrones de implicación, que se dan a partir de la potenciación y coordinación de redes territoriales, y su alteración morfológica a partir de su descentralización y territorialización de su esquema de intervenciones. Lo que realmente reditúa al Estado es la calidad y fortaleza de las estructuras estatales que operan en el nivel regional; esto es, los legados políticos y los aprendizajes históricamente desarrollados.

La construcción de capacidades estatales, entonces, se da por la fortaleza adquirida de sus instituciones y las redes de articulación históricamente desarrolladas por el Estado con los actores económico-sociales. En tal sentido, el Estado regional obtendrá autonomía a partir de sus sólidas capacidades internas.

Sin embargo, dichas capacidades no se encuentran en la totalidad de las estructuras estatales, ya que con base en las áreas de decisión estratégica por función, el área económica sobre el sector industrial es la más relevante, del mismo modo y de acuerdo con los niveles de acción sistémica, es en el nivel meso, ${ }^{1}$ donde se crean las condiciones propicias para la organización colectiva de las instituciones, de manera que puedan incidir directamente en los legados, en los modos de cooperación y de reproducción que requieren las nuevas condiciones de acumulación flexible.

De igual manera, en esta primera parte se integra el marco metodológico que sirve de base en la fase empírica posteriormente desarrollada. Se profundiza en los principios sobre capacidades internas y capacidades infraestructurales. Respecto a las internas, se muestran las articulaciones entre las OEPER a partir de tres grandes campos: el hardware (evaluación cuantitativa y articulada de los recursos financieros); el software (los re-

${ }^{1}$ Para Messner, la dinámica del desarrollo económico en este caso industrial, no sólo es sobre la base del funcionamiento de las políticas de mercados (nivel macro) y la iniciativa empresarial individual (nivel micro), sino también los esfuerzos colectivos para dar forma a un entorno propicio para el desarrollo integral. 
cursos humanos) y el orgware (el modelo de organización interna). Respecto a las capacidades infraestructurales, se enfocan al enraizamiento de las agencias estatales regionales, y el desarrollo de las acciones para definir procesos dinámicos de interacción sinérgica con los actores económicos y sociales desarrollados en el ámbito territorial (p. 63).

Por su parte, la segunda vertiente muestra un estudio de caso fundamentado temporalmente a partir de la década de los noventa y, espacialmente aplicado en el nivel mesorregional en el sector industrial en la provincia de Santa Fe. En esta fase empírica, se desarrolla una investigación centrada en las capacidades estatales y en la introducción de políticas macro, a partir del Consenso de Washington, y se analiza cómo han evolucionado en Argentina las capacidades estatales de las oeper, que articulan la producción, el territorio y la industria, así como la organización colectiva de los actores públicos y privados en el ámbito regional.

En tal sentido, y precisando el objeto de la investigación, la provincia de Santa Fe se posiciona como una importante representatividad económica en el contexto argentino, aunado a las características que, específicamente, han marcado la apertura económica con reformas estructurales orientadas al retiro y reducción del Estado. Con base en la parte metodológica presentada en la primera vertiente, se realiza un análisis de las oficinas que se presentan como estratégicas en las estructuras estatales, determinando las funciones de cada una las áreas de acción: área de acumulación económica, social o de legitimación y área de gobierno. Cabe señalar que estas oficinas pertenecen al nivel mesorregional, lo que permite coordinar acciones con los actores regionales y locales (p. 83).

Posterior a la determinación de los niveles de acción sistémica, áreas estatales y oficinas prioritarias, se muestra la secuencia del análisis referido a las capacidades internas. El hardware desde una visión holística, verifica las características de los recursos endógenos y exógenos, así como su distribución en las diferentes áreas del Estado; el software evalúa el reclutamiento, la forma de ingreso del personal, el tipo y nivel de formación educativa; y en el orgware se estiman cualitativamente los patrones de organización, la capacidad de decisión para formular ideas autónomas y el performance de las oficinas en términos de producción.

La autonomía lograda por el Estado regional, justamente a partir del desarrollo de las capacidades internas, requiere complementarse a su vez con las capacidades de infraestructura. En el caso de estudio, se analiza la gama de recursos económicos implicados en las acciones de enraizamiento, su distribución y alcance geográfico y las características que asumen las acciones de las OEPER, lo que permite identificar tanto actores como articulaciones para consolidar la interacción sinérgica de redes coopera- 
tivas, que para los autores pueden ser complementarias a las actividades de acuerdo con el área de acumulación flexible.

Después de un amplio y profundo análisis cuantitativa y cualitativamente desarrollado de las categorías, variables e indicadores, que muestran las capacidades estatales en la dinámica productiva regional de la provincia de Santa Fe, durante el periodo definido, se concluye que el Estado en sus distintas escalas y mediante sus diversas articulaciones, es un actor central y esencial para materializar estrategias de desarrollo regional y local; sin embargo, se presenta una debilidad en cuanto a la construcción de una sólida capacidad interna y la obtención de un adecuado enraizamiento, aunque se destaca la centralidad del nivel mesorregional del Estado como la instancia-motor de los nuevos patrones de organización, basados en redes y con capacidad de impulsar procesos productivos e innovadores ante las necesidades y desafíos que pudieran presentarse.

Finalmente, en la tercera vertiente se evalúan los resultados obtenidos en la primera y segunda partes del trabajo y se formula un conjunto de propuestas que buscan recuperar al Estado regional, vía la construcción de capacidades institucionales para superar el fragmentalismo y, de esta manera, alcanzar estrategias de desarrollo integral.

Partiendo de la pregunta planteada al inicio de la presente reseńa, la participación del Estado deriva, entonces, del reforzamiento de una estatidad con altas capacidades en áreas vinculadas a la producción industrial, pero la potencialidad de estas áreas necesariamente debe estar impulsada por aquéllas ligadas a la educación, la salud y la gestión gubernamental, es decir, la construcción de capacidades estatales como objetivo principal no se debe concebir en forma sectorialmente aislada. En tal sentido, se posiciona el nivel mesorregional como la unidad territorial adecuada para impulsar y articular la organización de los diferentes actores sociales en el desarrollo. No obstante, la colocación de la estatidad mesorregional como instancia fundamental destinada a evitar tanto el fragmentalismo localista como las asimetrías, no se puede concebir aisladamente respecto a las instancias nacionales donde se configura también una alta calidad institucional. De esta manera, y para explicitar la construcción de capacidades estatales mesorregionales como objetivo estratégico, resulta primordial recurrir a los horizontes de planeación, sobre todo a mediano y largo plazos.

La última parte del texto resulta sustancialmente interesante, porque además se formula un conjunto de lineamientos propositivos como tareas prioritarias en la construcción y consolidación de capacidades, tanto internas como de infraestructura, que abren la posibilidad de que la estatidad regional se transforme en un factor fundamental de impulso a los procesos 
de desarrollo territorial, pero condicionada al despliegue de una dinámica interactiva plena con los actores económico-territoriales (p. 235).

La investigación, sin lugar a dudas, hace un llamado al debate territorial y permite indagar una amplia discusión respecto a dos aspectos de particular importancia: el primero relacionado con la estructura teóricaconceptual que no sólo define la recolocación del Estado como motor del desarrollo regional, sino además profundiza en la forma en que éste debe participar en las diferentes instituciones y áreas estratégicas, potenciando la generación de capacidades en aquellas instancias de menor desarrollo, al tiempo que se inserta en un cuadro de articulación institucional multiescalar. Del mismo modo, el debate queda abierto a un replanteamiento de la perspectiva regional, insistiendo en instrumentos teórico-alternativos que permitan avanzar en la explicación y revertir las desigualdades regionales ante los nuevos escenarios, constituidos en un contexto globalizador y ante las repercusiones de los paradigmas técnico-económicos.

El segundo relacionado con el trabajo empírico, es decir, el estudio de caso seleccionado, refleja adecuadamente la disyuntiva entre el discurso de la descentralización y la promoción del desarrollo local endógeno y la realidad propiamente dicha. No obstante, los argumentos analizados en el texto se pueden profundizar, como sugieren los autores sobre estudios comparados en otras regiones de América Latina, y reflexionar sobre sus condiciones de partida, teniendo en cuenta las particularidades propias de cada caso.

\section{Bibliografía}

Fernández, Víctor (2001), Estrategias de desarrollo y transformación estatal. Buscando al Estado bajo el capitalismo global, Universidad Nacional del Litoral, Santa Fe.

Esser, Klaus, Wolfgang Hillebrand, Dirk Messner y Jörg Meyer-Stamer (1990), "Competitividad sistémica", www.meyer-stamer.de/ profil.html.

Messner, Dirk (1997), The Network Society. Economic Development and International Competitiveness as Problem of Social Governance, Frank Cass, Londres.

Recibido: 21 de julio de 2009. Aceptado: 30 de octubre de 2009. 
Rebeca Sesmas-Fragoso

Universidad Autónoma del Estado de México

Correo-e: rebecasesmas8@hotmail.com

Rebeca Sesmas-Fragoso. Cursa el doctorado en ciencias sociales en la Facultad de Ciencias Políticas y Sociales de la UAEM, desarrolla su tesis de grado en la línea de investigación de estudios socioterritoriales; es maestra en estudios urbanos y regionales y licenciada en planeación regional por la misma universidad. Ha sido colaboradora de investigación en diferentes espacios académicos de la dicha institución y profesor en unidades de aprendizaje vinculadas a: planeación ambiental, sociología, redes sociales, desarrollo local y sustentabilidad. Ha dirigido tesis en relación con las temáticas sobre estudios regionales/locales y ambientales. 